\title{
EchoGéo
}

15 | 2011

La Belgique, État prospère en décomposition au cœur de l'Europe du nord-ouest

\section{Une macro-géographie du commerce de détail en Belgique}

Jean-Pierre Grimmeau, Benjamin Wayens et Emilie Hanson

\section{(2) OpenEdition}

Journals

Édition électronique

URL : https://journals.openedition.org/echogeo/12330

DOI : $10.4000 /$ echogeo. 12330

ISSN : 1963-1197

Éditeur

Pôle de recherche pour l'organisation et la diffusion de l'information géographique (CNRS UMR 8586)

Référence électronique

Jean-Pierre Grimmeau, Benjamin Wayens et Emilie Hanson, « Une macro-géographie du commerce de détail en Belgique », EchoGéo [En ligne], 15 | 2011, mis en ligne le 12 avril 2011, consulté le 03 août 2021. URL : http://journals.openedition.org/echogeo/12330 ; DOI : https://doi.org/10.4000/echogeo. 12330

Ce document a été généré automatiquement le 3 août 2021

EchoGéo est mis à disposition selon les termes de la licence Creative Commons Attribution - Pas d'Utilisation Commerciale - Pas de Modification 4.0 International (CC BY-NC-ND) 


\title{
Une macro-géographie du commerce de détail en Belgique
}

\author{
Jean-Pierre Grimmeau, Benjamin Wayens et Emilie Hanson
}

\section{Introduction : quantifier et localiser le commerce de détail}

1 La géographie du commerce de détail a surtout été approchée en Belgique à partir des statistiques d'emploi (ou d'établissements) par commune (Mérenne-Schoumaker B., 1998b, Grimmeau J.-P. \& coll., 2004). Les zones de chalandises ont été déterminées par enquêtes (Mérenne-Schoumaker B., 1998a). Le réseau urbain a été étudié à partir des fonctions présentes (Van Hecke, 1998) et des navettes (Van Hecke E. \& coll., 2009). Les relevés de terrain, très consommateurs en temps, n'ont généralement servi qu'à des monographies urbaines (par exemple Mérenne-Schoumaker B. et Browet A., 1994; Wayens B., 2006). Le relevé qui s'est le plus approché d'une vue d'ensemble s'est limité aux noyaux de plus de 90 commerces des Régions urbaines ${ }^{1}$, soit quand même 40.000 cellules commerciales (Grimmeau, J.-P. \& coll., 2004). Par ailleurs, la localisation des centres commerciaux est bien connue, de même que le grand commerce (MérenneSchoumaker B., 1998b), à partir des relevés du SPF${ }^{2}$ économie, limités d'abord à l'alimentaire (des superettes aux hypermarchés) puis étendu aux principaux secteurs d'enseignes $^{3}$ de grande surface. D'autres études ont utilisé des adresses, soit d'enseignes, relevés principalement sur les sites internet (Grimmeau J.-P. \& Wayens B., $2000,2005)$, soit de tout le commerce d'une ville à partir d'annuaires à différentes dates (Grimmeau J.-P., Leroux V., Wayens B., 2007).

2 Le commerce de détail semble un concept clair : il regroupe tous les établissements vendant des biens ou des services aux particuliers et qui se distinguent généralement par une vitrine ou une enseigne ${ }^{4}$. Dans la classification NACE $^{5}$, suivie par la plupart des statistiques, le commerce de détail correspond au code 52 , beaucoup plus restrictif. Il manque par exemple l'HORECA ${ }^{6}$ (229000 actifs en 2000 d'après nos estimations; Grimmeau J.-P. \& coll., 2004), le secteur de l'automobile (127 000), les services (116 000), 
le secteur financier (où il ne faut considérer que les agences), etc., ensemble $44 \% \mathrm{du}$ commerce (1 060000 actifs). C'est néanmoins cette définition restreinte au code NACE 52 qui est généralement suivie par les économistes et les comparaisons géographiques intersectorielles, les géographes du commerce respectant la première.

Même si l'on règle le problème de la définition, l'emploi dans le commerce de détail ne peut faire l'objet que d'estimations. Par exemple, l'ONSS $^{7}$ ne renseigne que le personnel salarié. Il est possible, par comparaison avec les statistiques de la TVA, de déterminer le nombre d'établissements n'employant pas de personnel salarié. Mais combien de personnes travaillent dans ceux-ci (le patron, éventuellement le conjoint et/ou un ou plusieurs apparentés) ? Il n'est pas possible de trancher car les statistiques relatives aux indépendants (issues de l'INASTI ${ }^{8}$ ) ne sont pas ventilées dans la nomenclature NACE. De même, dans les établissements employant du personnel salarié, le chef d'établissement est compté s'il est salarié (gérant) mais pas s'il est indépendant (franchisé ou non). Enfin, une partie des travailleurs du secteur sont à temps partiel et les données en équivalents temps pleins sont rares. Ces différentes difficultés peuvent mener à des conclusions contradictoires selon les sources ou définitions choisies; on peut par exemple affirmer selon le cas que l'emploi dans le secteur du commerce de détail est en croissance ou en décroissance !

Ces circonstances expliquent l'intérêt des données produites en Belgique depuis 2006-2007 par la firme privée Locat'us. Il s'agit de relevés de terrain, répétés annuellement dans les zones commerciales denses, tous les deux ans ailleurs, qui enregistrent, pour chaque commerce : son type suivant une nomenclature très fine (226 catégories ${ }^{9}$ ), sa surface nette et l'enseigne éventuelle. Nous avons obtenu les données de 2008-2009 au niveau des codes postaux belges et l'objectif de cet article est de les analyser. La confrontation des données Locat'us avec d'autres données en notre disposition montre une bonne cohérence générale.

5 Deux réserves doivent cependant être faites. La première est que Locat'us est une firme néerlandaise et qu'elle ne suit pas toujours la nomenclature classique. Ainsi par exemple, le seuil considéré entre les superettes et les supermarchés est de $150 \mathrm{~m}^{2}$ et non de 400 comme il se doit (Dancette J. et Réthoré C., 2000 ; Desse R.-P. \& coll., 2008). D'autre part, les hypermarchés sont compris dans la catégorie grandes surfaces, qui comprend entre autres aussi les grands magasins (department stores), qui en Belgique se limitent maintenant à la seule enseigne Galeria Inno. Enfin, les surfaces n'ont pas été estimées en dehors du code NACE 52; nous ne disposons que des effectifs pour l'HORECA, l'automobile, les services et le secteur financier.

6 Les superettes, supermarchés, hypermarchés et grands magasins ont été redéfinis à partir du dernier inventaire du grand commerce par le SPF économie, qui date de 2008. Les surfaces des commerces absentes chez Locat'us ont été posées arbitrairement égales à $60 \mathrm{~m}^{2}$.

7 Les statistiques sur le commerce sont généralement fournies par communes. Celles-ci sont, depuis 1977, plus grandes en Belgique qu'en France par exemple : $50 \mathrm{~km}^{2}$ contre 15 en moyenne. Trois dépassent les $200 \mathrm{~km}^{2}$ : Tournai $\left(214 \mathrm{~km}^{2}\right)$, Couvin $\left(207 \mathrm{~km}^{2}\right.$ et Anvers $\left(205 \mathrm{~km}^{2}\right)$. Les statistiques par codes postaux permettent d'avoir une vision géographique plus fine (près de deux fois plus de codes postaux utiles que de communes) et plus homogène (les tailles de codes postaux, liées à l'organisation de la distribution spatiale du courrier, varient moins). 
8 Notre analyse sera essentiellement inductive, appuyée sur la construction de tableaux, de cartes et sur les techniques d'analyse des données.

\section{Le commerce de détail en Belgique}

9 Il y a en 2008-2009 en Belgique 194759 commerces (dont 89569 pour le seul code 52) pour une population de 10753080 habitants, soit 18 commerces par 1000 habitants $(8,3$ pour le seul code 52). La Belgique est connue pour sa forte densité commerciale. Au début des années 1990, la Belgique comptait un rapport commerces/habitants (14\%) proche de celui de l'Espagne et qui, dans l'Europe des douze n'était dépassé que par le Portugal, la Grèce et l'Italie (13 à 19\%o), les autres pays s'inscrivant entre 8 et $12 \%$ o (Eurostat 1993). La même observation avait déjà été faite par Jeanneney J.-M. (1954). Curieusement, de Kermadec C. \& Solard G. (2009) donnent pour la Belgique, en 2006, 6,7 entreprises commerciales pour 1000 habitants, soit une valeur proche de la France, à la limite supérieure de l'Europe du Nord-Ouest mais largement en-dessous des quatre pays méditerranéens cités précédemment. Bien sûr, il s'agit des entreprises et non des établissements mais cette différence ne suffit pas à expliquer l'écart par rapport à notre valeur de 8,3 .

Le tableau 1 présente les 20 types de commerces les plus fréquents et le tableau 2, les 20 types comptant la plus forte proportion d'enseignes ( $17 \%$ en moyenne). La surface commerciale totale est de $17772289 \mathrm{~m}^{2}$ si l'on s'en tient au code 52, pour laquelle elle est connue. La surface moyenne par commerce est alors de $198,4 \mathrm{~m}^{2}$, en fait 477 pour les enseignes et 138 pour les autres magasins. Le tableau 3 présente les 20 types de commerces de plus grande surface moyenne et les plus petits (code NACE 52). Toutes ces valeurs sont non seulement inédites pour la Belgique mais rares de façon générale. 
Tableau 1 - Les 20 types de commerces les plus fréquents en Belgique

\begin{tabular}{|lr|}
\hline Type de commerce & Effectif \\
\hline café & 15076 \\
coiffure & 13276 \\
restaurant & 10145 \\
boulangerie-pâtisserie & 6376 \\
agence de banque & 5754 \\
fast-food & 5303 \\
pharmacie & 5085 \\
café-restaurant & 4781 \\
prêt-à-porter féminin & 4736 \\
intermédiaire financier & 4618 \\
auto-mécanique & 4448 \\
boucherie-charcuterie & 3999 \\
tabac-journaux & 3726 \\
salon de beauté & 3593 \\
alimentation générale & 3558 \\
concessionnaire automobile & 3201 \\
vente de carburants & 3166 \\
prêt-à-porter (généraliste) & 2989 \\
lunchroom & 2922 \\
fleuriste & 2863 \\
\hline Source: Locat'us, 2009. & \\
\hline
\end{tabular}

Tableau 2 - Les 20 types de commerces où la part des enseignes est la plus importante

\begin{tabular}{|lr|}
\hline Type de commerce & \% d'enseignes \\
\hline & \\
hypermarché & 100,0 \\
grand magasin & 100,0 \\
agence de banque & 98,4 \\
supermarché $\left(+400 m^{2}\right.$ ) & 95,0 \\
prêt-à-porter (grande surface) & 90,3 \\
agence d'intérim & 86,5 \\
vente de carburants & 83,2 \\
surgelés & 82,8 \\
prêt-à-porter (libre-service) & 82,6 \\
paris sportifs & 74,2 \\
droguerie & 73,8 \\
bricocenter (+800m ${ }^{2}$ ) & 71,9 \\
meubles-ameublement & 70,2 \\
intermédiaire financier & 66,9 \\
appareils auditifs & 65,5 \\
auto-parebrise & 61,8 \\
supérette (-400m ${ }^{2}$ ) & 60,9 \\
télécom & 59,8 \\
chocolatier & 49,5 \\
parfumerie & 49,2 \\
& \\
\hline Source: Locat'us, 2009. & \\
\hline
\end{tabular}


Tableau 3 - Les 20 types de commerces ayant les plus petites et les plus grandes surfaces moyennes.

\begin{tabular}{|lrlr|}
\hline Type de commerce & $\begin{array}{r}\text { Surface nette }\left(\mathrm{m}^{2}\right) \\
\text { moyenne }\end{array}$ & Type de commerce & $\begin{array}{r}\text { Surface nette }\left(\mathrm{m}^{2}\right) \\
\text { moyenne }\end{array}$ \\
\hline hypermarché & & & \\
grand magasin & 7711 & bas-chaussettes & 45 \\
meubles-ameublement & 7918 & tabac-journaux & 42 \\
bricocenter $\left(+800 \mathrm{~m}^{2}\right)$ & 2376 & café-thé & 42 \\
meubles & 2110 & magasin d'hôpital & 41 \\
polyvalent & 1337 & night-shop & 41 \\
jardinerie & 1257 & poissonnerie & 39 \\
supermarché $\left(+400 \mathrm{~m}^{2}\right)$ & 1087 & bijouterie & 37 \\
prêt-à-porter (grande surface) & 974 & tabac & 37 \\
jardin-mobilier & 892 & gibier-volaille & 37 \\
vannerie & 622 & appareils auditifs & 36 \\
sport-outdoor & 616 & confiserie & 36 \\
surgelés & 553 & chocolatier & 34 \\
salle de bains-cuisines équipées & 530 & pharmacie & 33 \\
équipement de la maison & 521 & boucherie-charcuterie & 32 \\
jouets-jeux & 493 & encre-toner & 32 \\
sport-général & 471 & horlogerie & 31 \\
salle de bains & 470 & fromager & 31 \\
matériaux de construction & 461 & boulangerie-pâtisserie & 30 \\
articles de ménage & 408 & monnaie-timbres & 28 \\
\hline
\end{tabular}

11 Pour fournir une vue d'ensemble de la géographie du commerce en Belgique, nous avons cartographié la densité commerciale ${ }^{10}$, soit le rapport entre la surface commerciale et la superficie totale du canton postal (carte 1). Cet indicateur a été préféré au rapport de la surface commerciale à la population, qui met trop en évidence les parcs commerciaux ${ }^{11}$, installés en périphérie, là où les densités de population sont plus faibles. La carte met d'abord en évidence un contraste Flandre-Wallonie. Elle rappelle en fait plus encore la carte de densité de population. La Flandre a presque partout une densité supérieure à 200 habitants au kilomètre carré, alors que la Wallonie a une densité généralement inférieure à 100, sauf dans l'axe urbain et industriel allant de Mons-Borinage à Verviers. C'est la densité de population qui apparait donc primordiale pour la répartition globale du commerce,. Le commerce est une activité induite. Les communes de moins de 50 habitants par kilomètre carré présentent très peu de commerces. 
Carte 1 - Densité commerciale par canton postal

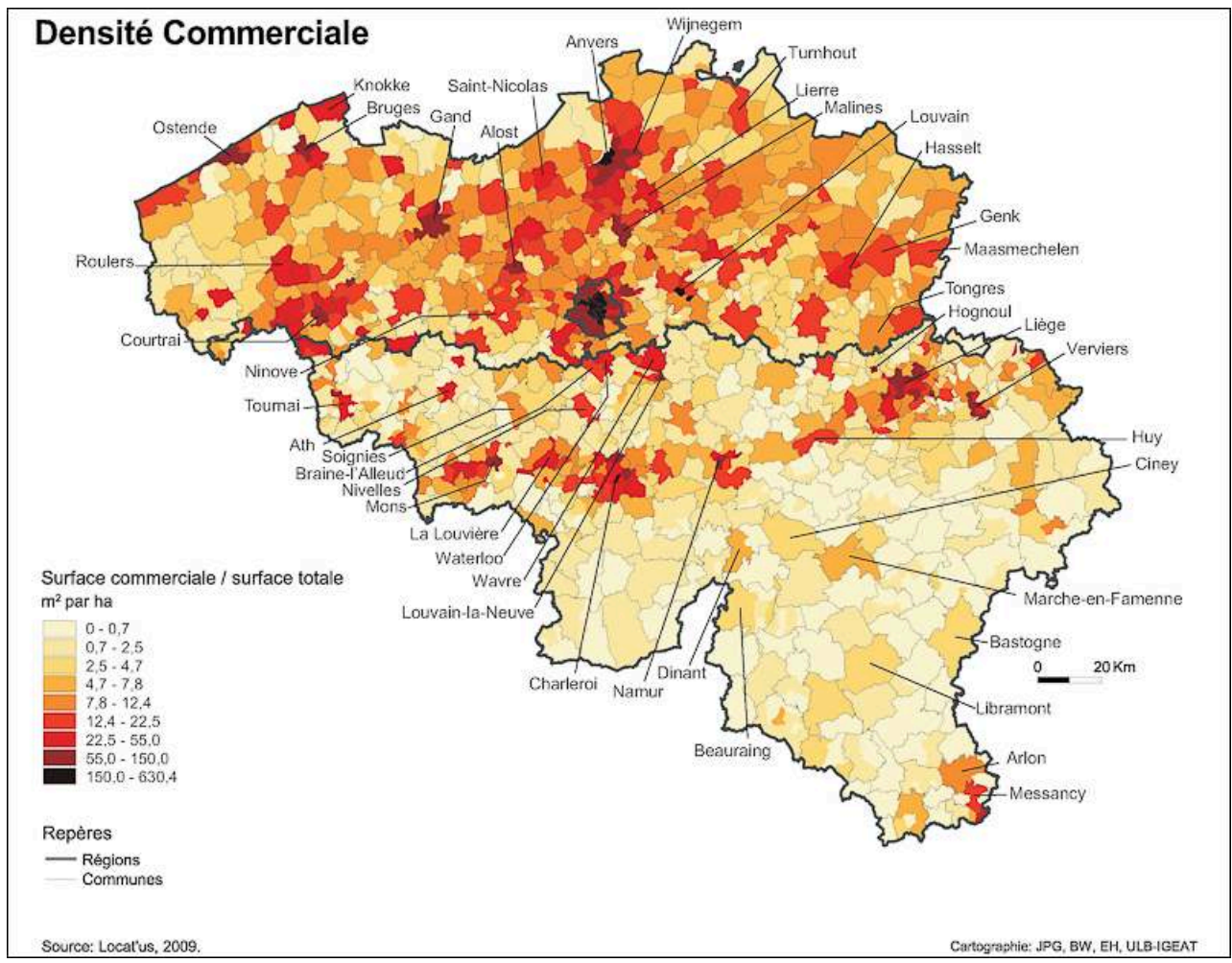

Source : Locat'Us, 2009 ; cartographie : J.-P. Grimmeau, B. Wayens, E. Hanson (ULB-IGEAT).

\section{Les composantes principales de la différentiation spatiale des structures commerciales}

Pour étudier les structures commerciales, une analyse en composantes principales a été réalisée sur la part de chaque type de commerce dans le total du code postal, en surface et en nombre de points de vente, pour les seules enseignes et pour tout le commerce; il y a donc quatre séries de pourcentages dont le total fait chaque fois 100. Les codes postaux ont été pondérés par la moyenne de leur importance relative par rapport au pays des quatre totaux (nombre et surface, des enseignes et de tout le commerce).

La première composante principale place du côté positif le prêt-à-porter, en particulier féminin, la parfumerie, les accessoires de mode, la lingerie, les restaurants du midi, la téléphonie, la maroquinerie et les disquaires, bref ce qui fait l'essentiel des rues les plus commerçantes, les plus passantes. Sont opposés, du côté négatif, les banques, boulangeries, stations d'essence, les garages, les supermarchés, les intermédiaires financiers, les coiffeurs et les boucheries, soit pour l'essentiel des types de commerces proches de la population.

Si l'on compare les scores avec la hiérarchie urbaine (Van Hecke E., 1998), on trouve évidemment parmi les plus fortes valeurs positives les cinq grandes villes (dans l'ordre décroissant des scores : Bruxelles, Anvers, Charleroi, Liège et Gand). Mais la hiérarchie commerciale ne se calque plus aujourd'hui seulement sur celle des villes, et deux localités dotées de grands centres commerciaux, Louvain-la-Neuve (dans le Brabant wallon) et Wijnegem (dans la banlieue anversoise), s'intercalent entre les trois 
premières villes ${ }^{12}$. Plusieurs villes régionales et une petite ville se placent entre Charleroi et Gand : Louvain, Namur, Knokke, Mons, Hasselt et Bruges. La petite ville de Knokke, station littorale la plus orientale, est aussi la plus huppée. S'additionnent ici les effets favorables du tourisme (voir Wayens B. \& Grimmeau J.-P., 2003) et du pouvoir $\mathrm{d}$ 'achat. Les autres villes régionales se dispersent dans des valeurs plus faibles mais toujours positives. On pourrait penser que, parmi les vingt villes régionales, celles qui viendraient en tête seraient les douze qui, avec leur banlieue, atteignent au moins 80000 habitants (concept de Régions urbaines, voir Van Hecke E. \& coll., 2009), mais il n'en est rien. Par exemple, Alost est ici mieux coté que Malines, et Turnhout que Verviers. Entre ces villes régionales s'intercalent des petites villes et des parties noncentrales de grandes villes. Une bonne partie des stations littorale apparaissent ici, comme aussi venant juste avant Verviers, Maasmechelen caractérisé surtout par son centre de magasins d'usine, construction contemporaine de type Disneyland sur un ancien site minier limbourgeois. Ce village est constitué de magasins de marques de producteurs, vendant sous forte réduction les collections de la saison précédente. Le facteur prépondérant de localisation de ce genre d'infrastructure est l'éloignement par rapport aux principaux ensembles de commerces traditionnels, où ces mêmes marques font l'essentiel de leur chiffre d'affaire. La dernière ville régionale est Arlon, plus petite des villes régionales, située dans l'extrémité sud-est de la Belgique. Plus grande ville de la province de Luxembourg, la moins peuplée des provinces belges, elle en est le cheflieu, ce qui lui assure une série de fonctions et, en conséquence, le statut de ville régionale malgré sa faible population (moins de 23000 habitants). Les scores négatifs caractérisent principalement les espaces ruraux ainsi que quelques petites villes.

La seconde composante principale présente des plus fortes saturations du côté négatif. On y trouve les grandes surfaces de prêt à porter (standing moyen), les magasins de peinture, papier-peint, rideaux, les meubles et l'ameublement, les articles pour bébés, les hypermarchés, les magasins de surgelés, ceux de literie et chambres à coucher, les magasins de chaussures, les magasins d'articles de bricolage de plus de $800 \mathrm{~m}^{2}$, les articles de ménage, les magasins de sports, ... Du côté positif, les types les mieux corrélés sont les cafés, les restaurants, les coiffeurs, les pharmaciens, les agences de banque, les tabac-journaux, les boulangeries-pâtisseries, les fast-foods, les restaurants du midi, les boucheries-charcuteries, ... Bref, il s'agit d'une opposition entre les espaces périphériques, dotés de parcs commerciaux, et les espaces urbains denses, caractérisés par des commerces desservant essentiellement la population locale. Ces deux premières composantes correspondent parfaitement à celles que nous avions obtenues par une analyse de la structure des enseignes (Grimmeau J.-P. et Wayens B., 2000). Il semble bien que l'on ait là les oppositions fondamentales en termes de localisation commerciale, aussi bien pour les enseignes que pour l'ensemble du commerce.

La troisième composante principale présente une assez belle opposition FlandreWallonie, Bruxelles et, dans une certaine mesure, Anvers présentant des scores positif comme en Wallonie. Cette opposition Flandre-Wallonie ressort encore mieux dans l'étude des enseignes (Grimmeau J.-P. \& Wayens B., 2000, 2005).

La quatrième composante présente des saturations négatives avec l'équipement de la maison, les cadeaux-souvenirs, les antiquaires, la HiFi-video, les magasins de monnaies et de timbres de collection, des magasins d'articles de sport très spécialisés, des bouquinistes, des grands magasins (Galeria Inno), ... , bref des types de commerces ayant besoin de zone de chalandise étendues, caractéristiques des grandes villes, mais, 
pour certains, plutôt des marges de l'hypercentre (voir Grimmeau J.-P.\& coll., 2004). Les types de commerce corrélés positivement n'ont pas besoin de zones de chalandise aussi étendues : drogueries \& soins, libres-services de prêt-à-porter, opticiens, nettoyages à sec, videothèques, matériels photo-video, articles de ménage, supermarchés, cordonneries-clefs, ... On rencontre ici une série de commerces de base des centrescommerciaux.

Comme nous l'avons déjà montré dans le passé à différentes échelles (Grimmeau J.-P. \& coll., 2004, Wayens B., 2006), les grandes catégories, fréquemment utilisées en géographie commerciale, sont hybrides sur le plan des logiques spatiales. Limitonsnous ici à quelques exemples de liaison à la première composante. Si l'alimentaire est généralement au pôle proximité de la composante, les chocolats sont au pôle des rues commerçantes. Dans les soins personnels, les pharmaciens s'opposent de même aux parfumeurs. Dans l'HORECA, les cafés et fast-foods sont de proximité mais les restaurants sont centraux. L'avantage de l'analyse en composantes principales est de positionner les catégories indépendamment de la classification, des lieux et du temps (nous avons aussi montré que certaines catégories "changent de côté" au cours du temps ; Grimmeau J.-P., Leroux V., Wayens B., 2007).

\section{Une typologie des lieux selon la structure commerciale}

19 Plutôt que d'entrer davantage dans l'analyse de ces composantes, d'autant plus délicate à mesure que le rang des composantes augmente, nous avons réalisé une typologie selon le critère de Ward (minimisation de la variance intra-groupes, maximisation de la variance inter-groupes) sur la base des scores non réduits des quatre premières composantes et en pondérant les observations de la même façon que pour l'ACP. La méthode de Ward a été poussée jusqu'à ne garder que 10 groupes. Après leur examen attentif, nous avons décidé de regrouper trois types correspondant à l'offre de plus grand rayonnement. Nous gardons donc huit groupes (carte 2). Ces types pourraient être améliorés par analyse de transfert mais nous y avons renoncé faute de temps. Pour chacun, nous avons calculé la densité commerciale $\left(\mathrm{m}^{2} / \mathrm{ha}\right)$, le nombre de commerces pour 1000 habitants, la surface moyenne des commerces (dans le code 52) et la proportion d'enseignes. Nous examinerons ensuite les types de commerces les plus spécifiques. 
Carte 2 - Importance et typologie du commerce

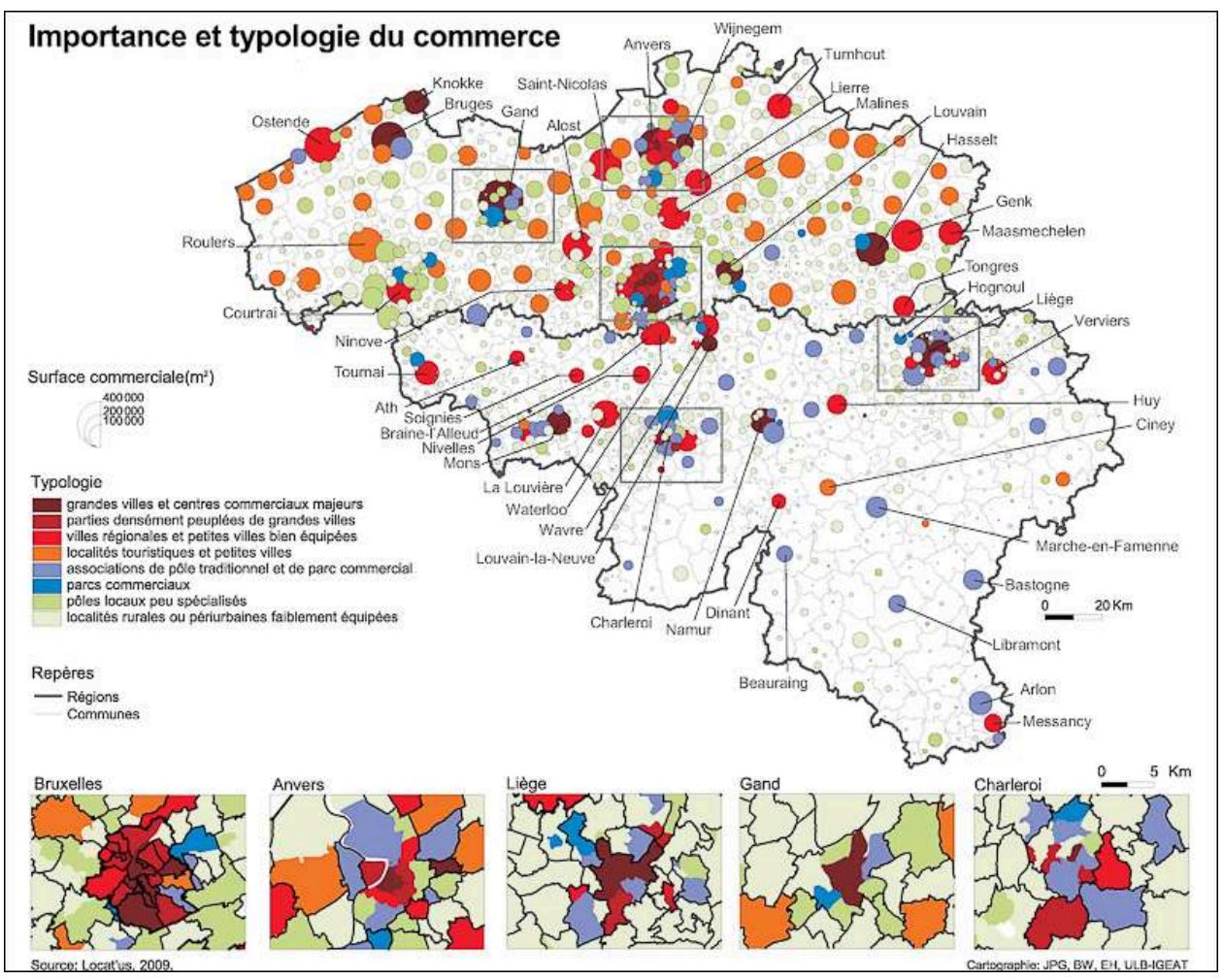

Source : Locat'Us, 2009 ; cartographie : J.-P. Grimmeau, B. Wayens, E. Hanson (ULB-IGEAT).

La carte montre l'abondance des villes régionales et des petites villes en Flandre alors qu'elles sont très rares au sud de l'axe Mons-Verviers. C'est évidemment une conséquence de la différence de densité de population. Par contre, les petites villes avec parc commercial ont la répartition inverse : la plus grande distance entre les villes est compensée par une offre moderne qui s'y juxtapose. 
Tableau 4 - Description des groupes de cantons postaux (typologie) : densité commerciale, nombre de commerces pour 1000 habitants, surface moyenne des commerces (dans le code 52) \& proportion d'enseignes

\begin{tabular}{|c|c|c|c|c|c|}
\hline Type de canton postal & $\begin{array}{r}\text { Densité } \\
\text { commerciale }\left(\mathrm{m}^{2}\right. \\
(\mathrm{ha}) \\
\end{array}$ & $\begin{array}{l}\text { Points de vente } \\
\text { par } 1000 \text { hab. }\end{array}$ & $\begin{array}{r}\text { Surface nette } \\
\text { moyenne } \\
\text { (NACE 52) }\end{array}$ & $\%$ enseignes & $\begin{array}{r}\text { Densité de } \\
\text { population } \\
\text { (hab./ha) }\end{array}$ \\
\hline $\begin{array}{l}\text { grandes villes et centres } \\
\text { commerciaux majeurs }\end{array}$ & 101 & 35,8 & 146 & 16.1 & 27 \\
\hline $\begin{array}{r}\text { parties densément peuplées de } \\
\text { grandes villes }\end{array}$ & 26 & 15,2 & 125 & 10,8 & 19 \\
\hline $\begin{array}{r}\text { villes régionales et petites villes } \\
\text { bien équipées }\end{array}$ & 32 & 24,3 & 208 & 20,9 & 10 \\
\hline $\begin{array}{r}\text { localités touristiques et petites } \\
\text { villes }\end{array}$ & 13 & 23,9 & 204 & 18,8 & \\
\hline $\begin{array}{l}\text { association pôle traditionnel et } \\
\text { parc commercial }\end{array}$ & 12 & 17,5 & 288 & 19,9 & \\
\hline parc commerciaux & 35 & 19,5 & 553 & 28,0 & 6 \\
\hline pôles locaux peu spécialisés & 9 & 17,5 & 218 & 15,9 & \\
\hline $\begin{array}{l}\text { localités rurales ou périurbaines } \\
\text { faiblement équipées }\end{array}$ & 3 & 13,6 & 187 & 14,9 & 2 \\
\hline tous types (profil moyen) & 8 & 18,9 & 198 & 16,9 & 3 \\
\hline
\end{tabular}

21 Le premier groupe réunit les parties les plus commerciales des grandes villes, cinq villes régionales, les mieux placées sur la première composante, Wijnegem et Louvainla-Neuve pour leur centre commercial et Knokke grâce au tourisme et au standing très élevé. Il réunit $16 \%$ des magasins et se distingue par une forte densité commerciale et par un nombre élevé de commerces par rapport à la population. La taille des commerces et la part d'enseignes sont inférieures à la moyenne.

Le deuxième groupe, $5 \%$ des commerces, correspond pour l'essentiel à des parties densément peuplées de grandes villes, où le commerce dessert surtout la population locale. La densité commerciale est élevée grâce à la densité de population; le nombre de commerces pour 1000 habitants est inférieur à la moyenne : la population est donc (légèrement) dépendante. C'est la catégorie dans laquelle la surface moyenne des commerces et la proportion d'enseignes sont les plus faibles.

Le troisième groupe, $14 \%$ des commerces, réunit dix villes régionales, des petites villes bien équipées, certaines favorisées par le pouvoir d'achat local (comme Waterloo, Braine-l'Alleud, dans la banlieue très aisée de Bruxelles) ou dotées d'un centre de magasins d'usine (Maasmechelen) et des communes non centrales de grandes villes. La densité commerciale est encore élevée de même que le nombre de commerces pour 1000 habitants. La surface moyenne des commerces est supérieure à la moyenne. La proportion d'enseignes est la deuxième plus forte.

Le quatrième groupe, $14 \%$ des commerces, comprend surtout des petites villes et des localités touristiques, principalement littorales. La densité commerciale est sensiblement plus faible que les précédents et le nombre de commerces pour 1000 habitants est au niveau du groupe 3. La taille des commerces et la part d'enseignes sont encore légèrement au-dessus de la moyenne.

Le cinquième groupe, $8 \%$, se compose de localités qui associent un parc commercial et du commerce traditionnel. Les densités de population ne sont pas négligeables: banlieues, couronnes urbaines dense ou petites villes. Arlon est aussi rangée dans cette catégorie. La densité commerciale et le nombre de commerces pour 1000 habitants 
sont proches de la moyenne. Par contre, les commerces sont plus grands et il y a plus d'enseignes.

Le sixième groupe, $1,2 \%$ seulement, concerne les localités dotées d'un parc commercial sans autre présence commerciale importante. La majorité de ces localités sont situées aux portes des grandes villes. La densité commerciale est légèrement supérieure à celle des grandes villes et le nombre de commerces pour 1000 habitants à la moyenne. C'est ici que les magasins sont les plus grands et les enseignes les plus fréquentes.

Le groupe 7, $15 \%$ du commerce, réunit dix petites villes, de la banlieue, des localités de couronne urbaine et du rural. Les quatre caractéristiques générales du septième groupe sont proches de la moyenne. La densité de population est moyenne et le commerce ne dessert guère plus que la population locale.

Enfin, le groupe 8, qui couvre la plus grande surface, le plus grand nombre de localités, et la plus grande part du commerce $(27 \%)$, présente les plus faibles densités commerciales et nombre de commerces pour 1000 habitants. La surface moyenne des commerces est légèrement inférieure à la moyenne. La proportion d'enseignes est la deuxième plus petite, après le type 2. Les communes sont ici majoritairement rurales quoiqu'il y ait aussi des localités de banlieue ou de couronne urbaine mais le commerce est purement local.

Pour définir les types de commerces caractéristiques de ces types d'espaces, nous avons déterminé par $\mathrm{Chi}^{2}$ ceux qui étaient surreprésentés significativement dans chaque groupe de codes postaux. Les tests statistiques ne pouvant s'appliquer qu'à des effectifs, nous avons donc travaillé sur les nombres de commerces. Les types caractéristiques des parcs commerciaux apparaissaient mieux en travaillant sur la somme des groupes 5 (petite ville + parc commercial) et 6 (parc commercial) et ceux des petites villes en ne considérant que le type 4 (petites villes). Il n'apparaissait pas d'ensemble cohérent de types de commerces pour les petites villes avec parc commercial ni pour le type périphérique. Pour attribuer chaque type de commerce à une seule catégorie, nous avons choisi parmi celles à surreprésentation significative, celle où la part de ce type de commerce dans le total des commerces du groupe de codes postaux était la plus élevée. Enfin, si la catégorie retenue était un des trois niveaux urbains, nous l'avons affectée au niveau le plus bas où la surreprésentation était significative. Le solde des types de commerces est classé "indifférents".

Les types de commerces caractéristiques les plus importants des grandes villes sont les restaurants, les bijouteries, les antiquaires, les magasins de porcelaines et cristaux et les chocolatiers. Dans le groupe urbain dense viennent l'alimentation générale, les pharmaciens, les centres de téléphonie et d'internet, le nettoyage à sec et les laveries automatiques ainsi que les tabacs-journaux. Pour les villes régionales, on a les restaurants du midi, les agences de voyage, les magasins de téléphones portables (GSM), les librairies et les magasins d'Hi-Fi \& video. Dans les petites villes apparaissent le prêt-à-porter féminin et général, les brasseries, les magasins de chaussures et les agences immobilières. Sont caractéristiques des parcs commerciaux les concessionnaires de voitures, les supermarchés, les magasins d'électroménager et multimédia, de meubles et les réparateurs auto spécialisés. Enfin, dans le commerce de base, les plus fréquents sont les cafés, les coiffeurs, les boulangers, les banques et les garages.

31 Le graphique (figure 1) montre comment ces sept groupes de commerces se répartissent entre les huit types de codes postaux. Ce sont dans les grandes villes que 
les quatre groupes de commerces urbains représentent la plus grande part cumulée (64\%). Cette part descend à $52 \%$ dans le type urbain dense non central, à $50 \%$ dans les villes régionales, à 45 ou $41 \%$ dans les petites villes (sans ou avec parc commercial) et entre 37 et $30 \%$ pour les trois dernières catégories de codes postaux. Les types de commerces caractéristiques des grandes villes représentent $17 \%$ des commerces du groupe des grandes villes, contre 8 à $10 \%$ ailleurs. C'est aussi dans le groupe grande ville que les types de commerces caractéristiques des villes régionales et petites est maximal: sont rangés dans les commerces caractéristiques des petites villes par exemple les types surreprésentés à partir de ce niveau mais qui peuvent évidemment avoir plus d'importance dans les villes régionales ou grandes. Les commerces caractéristiques des parties denses non centrales des villes représentent $27 \%$ des commerces du groupe correspondant contre 9 à $15 \%$ ailleurs. Les commerces caractéristiques des villes régionales représentent $8 \%$ des commerces des grandes villes, $6 \%$ dans les villes régionales et entre 2 et $4 \%$ ailleurs. Les commerces caractéristiques des petites villes forment $24 \%$ des commerces des grandes villes, $22 \%$ des villes régionales, $23 \%$ des petites villes et entre 10 et $15 \%$ ailleurs. Les commerces typiques des parcs commerciaux constituent $25 \%$ des commerces des lieux classés parc commercial et 17 de ceux classés petite ville avec parc commercial; entre 7 et $13 \%$ ailleurs. Enfin, les commerces de base forment $56 \%$ des commerces du groupe 8, $46 \%$ du groupe 7 et entre 26 (grandes villes) et $40 \%$ (petites villes) ailleurs.

Figure 1 - Répartition des sept groupes de commerces dans les huit types de codes postaux

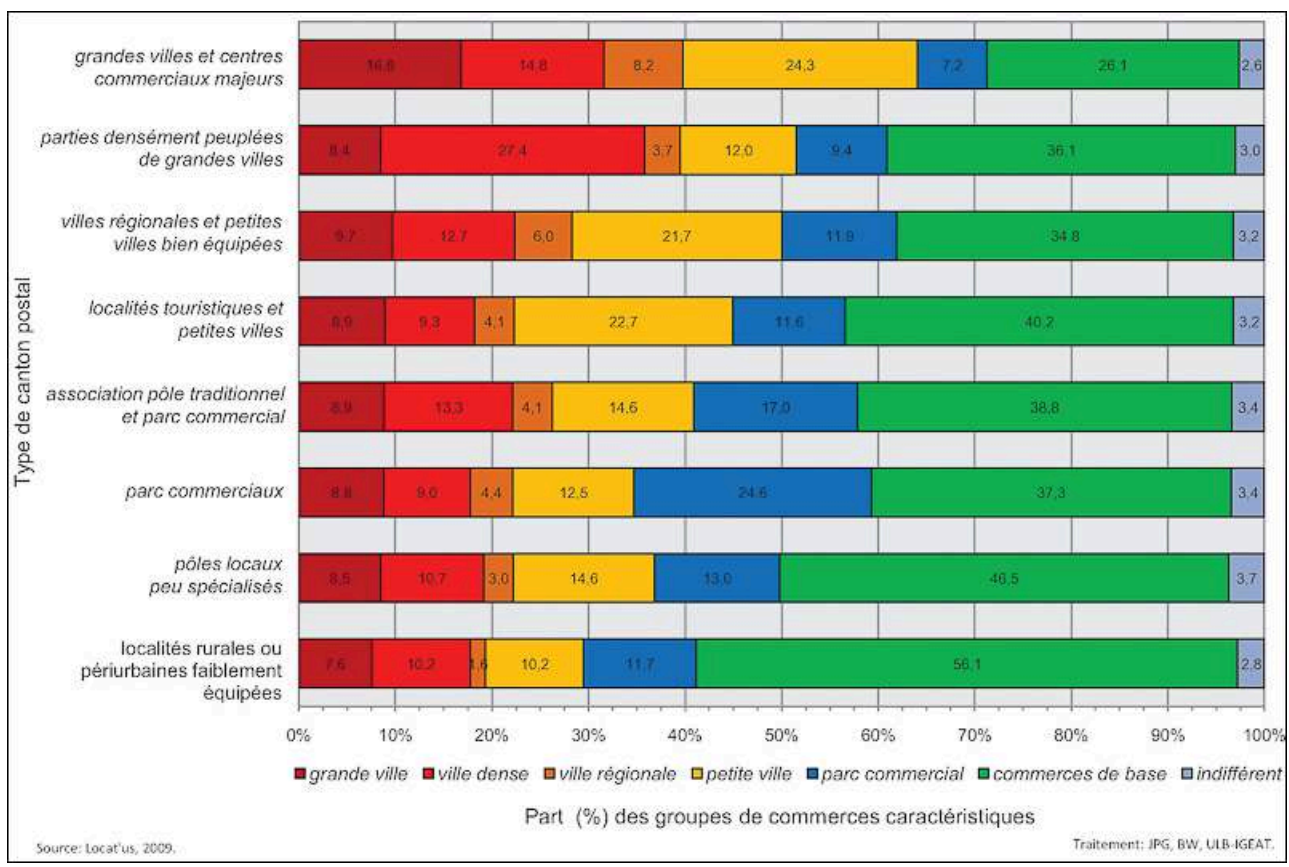

Source : Locat'us, 2009 ; traitement : J.-P. Grimmeau, B. Wayens (ULB- IGEAT).

\section{Conclusions}

1.- Les structures commerciales les plus diversifiées et les plus attractives ne sont plus l'apanage des grandes villes. Un pouvoir d'achat élevé ou l'attraction touristique peuvent soutenir un tel commerce dans des villes régionales voir petites. Les centres 
commerciaux majeurs peuvent aussi donner à une localité assez modeste un attrait commercial très important. Contrairement à notre attente, les localités à grand centre commercial ne se différencient pas par la structure.

2.- Contrairement à une idée parfois affirmée, il existe encore une différenciation sensible de structure commerciale entre les grandes villes, les villes régionales et les petites villes. Evidemment, la hiérarchie commerciale des villes en 2009 ne correspond pas strictement à la hiérarchie des villes telle que définie sur des données du début des années 1990 prenant en compte un ensemble diversifié de fonctions urbaines.

3.- Les parcs commerciaux constituent aujourd'hui des infrastructures commerciales importantes et nombreuses, susceptibles de compléter ou de suppléer l'offre commerciale existante. Les périphéries urbaines, où la population a crû plus vite que l'offre commerciale et où des terrains à bon compte étaient disponibles, sont une de leurs localisations privilégiées. Les régions à faible densité de population en sont une autre : tant qu'à devoir couvrir des distances importantes pour accéder au commerce, autant le regrouper.

\section{BIBLIOGRAPHIE}

Dancette J. \& Réthoré C., 2000. Dictionnaire analytique de la distribution. Les Presses de l'Université de Montréal, $347 \mathrm{p}$.

Desse R.-P. \& coll. (dir.), 2008. Dictionnaire du commerce et de l'aménagement. Presses universitaires de Rennes, $357 \mathrm{p}$.

Eurostat, 1993. Le commerce de détail dans le marché unique européen en 1993. Commission des Communautés Européennes, 213 p.

Grimmeau J.-P. (dir.), 2004. Le commerce des grandes villes belges et leurs périphéries. IGEAT, 147 p. Téléchargeable.

Grimmeau J.-P. \& Wayens B., 2000. Logiques spatiales des enseignes commerciales en Belgique. Gondola, 60, p. 20-25.

Grimmeau J.-P., Wayens B., 2005. Le facteur culturel dans les politiques de localisation : le cas belge. In Dioux J. \& Dupuis M. (ed.). La distribution. Stratégies des groupes et marketing des enseignes, Pearson-éducation, Paris, p. 252-253.

Grimmeau J.-P., Leroux V. \& Wayens B., 2007. Un demi-siècle d'évolution du commerce de détail à Bruxelles. Observatoire du commerce, Région de Bruxelles-capitale, Administration de l'Aménagement du Territoire et du Logement2, 75 p. Téléchargeable.

Jeanneney J.-M., 1954. Les commerces de détail en Europe occidentale. Armand Colin.

de Kermadec, C. \& Solard, G., 2009. Le commerce de détail en Europe. Diversité et convergence des tissus commerciaux. INSEE Première, $\mathrm{n}^{\circ} 1268$.

Mérenne-Schoumaker B., 1998a. Impact des mutations du commerce sur la hiérarchie des polarités commerciales, l'exemple de la Belgique. Hommes et Terres du Nord, 4, p. 217-224. 
Mérenne-Schoumaker B., 1998b. Centres de commerce de détail. Atlas de Belgique, Planche VIII.18, Commission de l'Atlas National.

Mérenne-Schoumaker B. \& Browet A., 1994. Atlas du commerce de détail de Liège. Ville de Liège, $99 \mathrm{p}$.

Van Hecke, E., 1998. Actualisation de la hiérarchie urbaine en Belgique. Bulletin du Crédit Communal, 3, p. 45-76.

Van Hecke E., Halleux J.-M., Decroly J.-M. \& Mérenne-Schoumaker B., 2009. Noyaux d'habitat et Régions urbaines dans une Belgique urbanisée. Monographies de l'enquête socio-economique 2001, $\mathrm{n}^{\circ}$ 9, SPF Economie. Téléchargeable.

Wayens B., 2006. Structure et dynamique du commerce de détail bruxellois. Thèse de doctorat inédite, ULB. Téléchargeable.

Wayens B. \& Grimmeau, J.-P., 2003. L'influence du tourisme sur la géographie du commerce de détail en Belgique. Belgeo, p. 289-302.

\section{NOTES}

1. Pour le concept de Région urbaine, voir Van Hecke \& coll., 2009.

2. Service Public Fédéral.

3. Au sens de réseau de points de vente.

4. Au sens de panneau signalant l'existence du commerce.

5. Nomenclature Européenne des Activités Economiques.

6. Acronyme utilisé en Belgique pour le secteur d'activités de l'Hôtellerie, de la Restauration et des Cafés. Son équivalent en France est CHR, pour Cafés-Hôtels-Restaurants.

7. Office National de la Sécurité Sociale.

8. Institut National d'Assurances Sociales pour Travailleurs Indépendants.

9. La nomenclature peut être obtenue sur demande auprès de Locat'us http://www.locatus.com

10. Les analyses préliminaires relatives à la densité commerciale ont été réalisées par P. MedinaLockhart dans le cadre de ses travaux pour la Conférence Permanente du Développement Territorial de la Région Wallonne.

11. Equivalent en Belgique des Zones d'Activités Commerciales en France.

12. Angleur (dans l'agglomération de Liège) arrive même en tête grâce au centre commercial de Belle-Ile, dont les commerces sont majoritairement attribués de façon erronée à ce code postal (80 commerces classés à Angleur pour 15 au code correct Liège2 et 1 à Liège1); les sites des enseignes donnent le code correct. Après correction, Angleur passe en $233 \mathrm{e}$ position. Tous les résultats publiés ici tiennent compte de cette correction.

\section{RÉSUMÉS}

Cet article analyse une nouvelle source, issue d'un inventaire de terrain exhaustif. Il confirme que la Belgique est dotée d'un équipement commercial très dense par comparaison aux pays d'Europe du nord-ouest. Il présente les types de commerces les plus fréquents, ceux qui comptent 
le plus d'enseignes, les plus grands et les plus petits en surface moyenne. La densité commerciale par codes postaux est cartographiée et les variations spatiales de la structure commerciale sont analysées au moyen d'une analyse en composantes principales et d'une typologie. Les résultats sont comparés à la hiérarchie urbaine. Le rôle du pouvoir d'achat et du tourisme est mis en avant, comme le développement des centres commerciaux et zones d'activités commerciales.

Thisarticleanalyzesanew source, followingacomprehensivefield inventory. ItconfirmsthatBelgiumhasadensecommercial equipmentcomparedto the countries of northwestern Europe. Itpresentsthemostnumerous types ofretail, those with the mostbanners, the largestandthe smallestinaverage size. Thecommercial densitybypostal codeis mappedandthe spatial variationsofthe commercial structureareanalyzedusingaprincipal components analysisandatypology. Theresultsarecomparedwiththe urban hierarchy.Theroleofpurchasing powerandtourismishighlightedasthedevelopment ofshopping centersandretail areas.

\section{INDEX}

Mots-clés : Belgique, commerce de détail, hiérarchie urbaine, pouvoir d'achat, tourisme, zone d'activités commerciales

Keywords : Belgium, purchasing power, retail, retail park, tourism, urban hierarchy

\section{AUTEURS}

\section{JEAN-PIERRE GRIMMEAU}

Jean-Pierre Grimmeau (grimmeau@ulb.ac.be) est professeur honoraire à l'Université Libre de Bruxelles (Faculté des Sciences - IGEAT).

\section{BENJAMIN WAYENS}

Benjamin Wayens (bwayens@ulb.ac.be) est maître de conférences et de chargé de recherche à l'Université Libre de Bruxelles (Faculté des Sciences - IGEAT).

\section{EMILIE HANSON}

Emilie Hanson (ehanson@ulb.ac.be) est assistante de recherche à l'Université Libre de Bruxelles (Faculté des Sciences - IGEAT). 